\title{
Supporting Information for Ionophilic Phosphines: Versatile Ligands for Ionic Liquid Biphasic Catalysis
}

Crestina S. Consorti*, Guilherme L. P. Aydos, Günter Ebeling, Jaïrton Dupont*

Laboratory of Molecular Catalysis, Institute of Chemistry - UFRGS, Av. Bento Gonçalves, 9500 P.O. Box 15003, 91501-970, Porto Alegre, RS Brazil

\section{General Remarks}

All manipulation of phosphine ligands and complexes were conducted under Ar using dryboxes or standard Schlenk techniques. Chemicals were treated as follows: ether, toluene, pentane, distilled from $\mathrm{Na}$ /benzophenone; $\mathrm{CF}_{3} \mathrm{Ph}$ (Alfa Aesar), distilled from $\mathrm{CaH}_{2}$; diethyl diallylmalonate (Lancaster); AIBN, ABCN, 1-vinyl-imidazole, 1,2-dimethylimidazole, allyl bromide, $\mathrm{LiNTf}_{2},\left(\left(1,3-\mathrm{Bis}(\mathrm{Mes}) \mathrm{H}_{2} \mathrm{Im}\right)\right)\left(\mathrm{PCy}_{3}\right)(\mathrm{Cl})_{2} \mathrm{Ru}(=\mathrm{CHPh})$ (Aldrich); $\mathrm{HPPh}_{2}, \mathrm{HPCy}_{2}$ (Strem); DMSO- $\mathrm{d}_{6}$, toluene- $\mathrm{d}_{8}$ (Cambridge Isotope) and other solvents, used as received.

Diethyl 2-allyl-2-methallylmalonate, ${ }^{1} \quad$ diethyl dimethallylmalonate, ${ }^{1} \quad((1,3-$ $\left.\operatorname{Bis}(\mathrm{Mes}) \mathrm{H}_{2} \mathrm{Im}\right)(\mathrm{Py})_{2}(\mathrm{Cl})_{2} \mathrm{Ru}(=\mathrm{CHPh}){ }^{2}$ ILs $\left(\mathrm{BMI}^{2} \mathrm{PF}_{6}, \mathrm{BMI} \mathrm{NTf}_{2}\right.$, and BMI.FAP), and $\mathrm{CH}_{3} \mathrm{SO}_{3} \mathrm{Bu}^{3}$ were synthesized according to literature procedures. NMR spectra were recorded on $300 \mathrm{MHz}$ Varian spectrometers at ambient probe temperatures and referenced to internal TMS or external $\mathrm{H}_{3} \mathrm{PO}_{4}$. GC analyses were performed using an Agilent 6820 instrument equipped with a capillary column (DB-17-0.25 mm; $25 \mathrm{~m} \mathrm{~S} 0.32 \mathrm{~mm}$ ). GC-MS data were recorded with a Shimadzu QP2010 and ESI/MS on a Micromass Q-TOFmicro. Elemental analyses were conducted at the central analitica/IQ-UFRGS. Ru content was measured on a PerkinElmer AAnalyst 200 atomic absoption spectrometer.

\section{1-allyl-2,3-dimethyl-imidazolium $\mathrm{NTf}_{2}(\mathbf{1})$}

A round bottom flask was charged with 1,2-dimethylimidazole (0.502 g, $52 \mathrm{mmol})$, allyl bromide $(6.41 \mathrm{~g}, 53 \mathrm{mmol})$ and acetonitrile $(50 \mathrm{~mL})$. The mixture was stirred at $60^{\circ} \mathrm{C}(5 \mathrm{~h})$. The solvent was removed by oil pump vacuum to give a brownish oil that was treated with activated carbon in boiling water $(100 \mathrm{~mL})$. After filtration, $\operatorname{LiNTf}_{2}(15.2 \mathrm{~g}, 53 \mathrm{mmol})$ was added in portions to the aqueous solution. The lower phase was diluted with $\mathrm{CH}_{2} \mathrm{Cl}_{2}(100$ 
$\mathrm{mL}$ ), dried over $\mathrm{MgSO}_{4}$ and solvent was removed by oil pump vacuum to give a yellowish oil; yield: $20.0 \mathrm{~g}(92 \%)$

${ }^{1} \mathrm{H}$ NMR (300 MHz, DMSO-d6) $\delta$ ppm 7.63 and $7.58\left(\mathrm{~d}, J^{3}=1.9 \mathrm{~Hz}, \mathrm{H} 4\right.$ and H5), $5.96(\mathrm{~m}$, $\left.\mathrm{NCH}_{2} \mathrm{C} \underline{H}=\mathrm{CH}_{2}\right), 5.29\left(\mathrm{~d}, J^{3}=10.4 \mathrm{~Hz}, \mathrm{NCH}_{2} \mathrm{CH}=\mathrm{CH}_{2}\right), 5.13\left(\mathrm{~d}, J^{3}=17.1 \mathrm{~Hz}\right.$, $\left.\mathrm{NCH}_{2} \mathrm{CH}=\underline{\mathrm{C}}_{2}\right), 4.80\left(\mathrm{~d}, J^{3}=5.4 \mathrm{~Hz}, \mathrm{NC} \underline{H}_{2} \mathrm{CH}=\mathrm{CH}_{2}\right), 3.75\left(\mathrm{~s}, \mathrm{NC} \underline{H}_{3}\right), 2.53\left(\mathrm{~s}, \mathrm{CC} \underline{H}_{3}\right)$. ${ }^{13} \mathrm{C}\left\{{ }^{1} \mathrm{H}\right\}$ NMR $\left(75 \mathrm{MHz}, \mathrm{DMSO}-d_{6}\right) \delta \mathrm{ppm} 144.5$ (s, C2), $131.5\left(\mathrm{~s}, \mathrm{NCH}_{2} \mathrm{CH}=\mathrm{CH}_{2}\right), 122.4$ and $121.0(\mathrm{~s}, \mathrm{C} 4$ and $\mathrm{C} 5), 119.4\left(\mathrm{q}, J_{\mathrm{CF}}=321.9 \mathrm{~Hz}, \mathrm{NTf}_{2}{ }^{-}\right), 118.8\left(\mathrm{~s}, \mathrm{NCH}_{2} \mathrm{CH}=\underline{\mathrm{CH}_{2}}\right), 49.6$ (s, $\left.\mathrm{NCH}_{2} \mathrm{CH}=\mathrm{CH}_{2}\right), 34.7\left(\mathrm{~s}, \mathrm{NCH}_{3}\right), 9.1\left(\mathrm{~s}, \mathrm{C} \underline{C} \mathrm{H}_{3}\right) . \operatorname{ESI} / \mathrm{MS}(+): \mathrm{m} / \mathrm{z} 137.08\left(\left[\mathrm{C}_{8} \mathrm{H}_{13} \mathrm{~N}_{2}\right]^{+}\right)$. Anal. Calcd for $\mathrm{C}_{10} \mathrm{H}_{13} \mathrm{~F}_{6} \mathrm{~N}_{3} \mathrm{O}_{4} \mathrm{~S}_{2}$ C 28.78; H 3.14; N 10.07. Found: C 28.74; H 3.13; N 10.01 .

1-vinyl-3-butyl-imidazolium $\mathrm{NTf}_{2}$ (2)

A round bottom flask was charged with 1-vinyl-imidazole $(9.41 \mathrm{~g}, 100 \mathrm{mmol})$ and $\mathrm{CH}_{3} \mathrm{SO}_{3} \mathrm{Bu}(15.2 \mathrm{~g}, 100 \mathrm{mmol})$. The mixture was stirred at $60^{\circ} \mathrm{C}(12 \mathrm{~h})$ yielding a viscous oil which was washed with ethylacetate $(3$ X $50 \mathrm{~mL})$. The resulting oil was diluted with water $(50 \mathrm{~mL})$ and $\operatorname{LiNTf}_{2}(30.1 \mathrm{~g}, 105 \mathrm{mmol})$ was added in portions. The lower phase was diluted with $\mathrm{CH}_{2} \mathrm{Cl}_{2}(100 \mathrm{~mL})$, dried over $\mathrm{MgSO}_{4}$ and solvent was removed by oil pump vacuum to give a yellowish oil; yield: $35.5 \mathrm{~g}(88 \%)$

${ }^{1} \mathrm{H}$ NMR (300 MHz, DMSO-d $) \delta$ ppm 9.46 (s, H2), 8.19 and 7.92 (s, H4 and H5), 7.27 (dd, $\left.J^{3}=15.6 \mathrm{~Hz}, J^{3}=8.7 \mathrm{~Hz}, \mathrm{NC} \underline{H}=\mathrm{CH}_{2}\right), 5.94\left(\mathrm{dd}, J^{3}=15.6 \mathrm{~Hz}, J^{2}=2.3 \mathrm{~Hz}, \mathrm{NCH}=\underline{\mathrm{H}}_{2}\right)$, $5.42\left(\mathrm{dd}, J^{3}=8.7 \mathrm{~Hz}, J^{2}=2.3 \mathrm{~Hz}, \mathrm{NCH}=\underline{\mathrm{C}}_{2}\right), 4.19\left(\mathrm{t}, J^{3}=7.22 \mathrm{~Hz}, \mathrm{NC} \underline{H}_{2}\right), 1.91-1.69(\mathrm{~m}$, $\left.\mathrm{NCH}_{2} \underline{\mathrm{CH}}_{2}\right), 1.41-1.19\left(\mathrm{~m}, \mathrm{NCH}_{2} \mathrm{CH}_{2} \mathrm{C}_{2}\right), 0.91$ (t, $\left.J^{3}=7.39 \mathrm{~Hz}, \mathrm{NCH}_{2} \mathrm{CH}_{2} \mathrm{CH}_{2} \mathrm{CH}_{3}\right)$. ${ }^{13} \mathrm{C}\left\{{ }^{1} \mathrm{H}\right\}$ NMR $\left(75 \mathrm{MHz}, D M S O-d_{6}\right) \delta$ ppm $135.2(\mathrm{~s}, \mathrm{C} 2), 128.8\left(\mathrm{~s}, \mathrm{~N} \underline{C H}=\mathrm{CH}_{2}\right), 123.2$ and $119.1\left(\mathrm{~s}, \mathrm{C} 4\right.$ and C5), $119.4\left(\mathrm{q}, J_{\mathrm{CF}}=321.9 \mathrm{~Hz}, \mathrm{NTf}_{2}^{-}\right), 108.5\left(\mathrm{~s}, \mathrm{NCH}=\underline{C H}_{2}\right), 48.9$ (s, $\left.\mathrm{N}_{\underline{C}} H_{2}\right), 31.0$ (s, $\left.\mathrm{NCH}_{2} \underline{\mathrm{C}} \mathrm{H}_{2}\right), 18.7$ (s, $\left.\mathrm{NCH}_{2} \mathrm{CH}_{2} \underline{\mathrm{CH}_{2}}\right), 13.2$ (s, $\left.\mathrm{NCH}_{2} \mathrm{CH}_{2} \mathrm{CH}_{2} \underline{C_{3}}\right)_{3}$. ESI/MS(+): m/z $151.12\left(\left[\mathrm{C}_{9} \mathrm{H}_{15} \mathrm{~N}_{2}\right]^{+}, 100 \%\right)$. Anal. Calcd for $\mathrm{C}_{11} \mathrm{H}_{15} \mathrm{~F}_{6} \mathrm{~N}_{3} \mathrm{O}_{4} \mathrm{~S}_{2} \mathrm{C} 30.63 ; \mathrm{H}$ 3.50; N 9.74. Found: C 31.08; H 3.31; N 9.83.

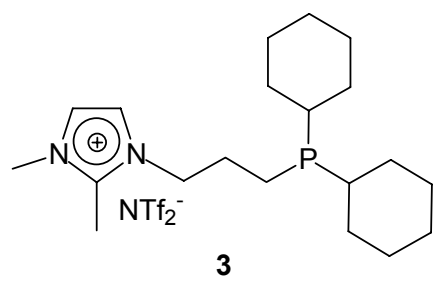

In a drybox, 1-allyl-2,3-dimethyl-imidazolium $\operatorname{NTf}_{2}(0.860 \mathrm{~g}$, $2.06 \mathrm{mmol}) \mathrm{HPCy}_{2}(0.793 \mathrm{~g}, 4.00 \mathrm{mmol}), \mathrm{CF}_{3} \mathrm{Ph}$ and $\mathrm{ABCN}$ 
(10 mg, $0.04 \mathrm{mmol}$ ) were combined in a resealable Schlenk flask. The mixture was stirred at $80{ }^{\circ} \mathrm{C}$ for 5 days; during which time 4 additional $\mathrm{ABCN}$ charges (48 $\mathrm{mg}, 0.20 \mathrm{mmol}$ ) were added to the reaction mixture. The volatiles were removed under vacuum and the resulting oil was washed with ethyl ether $(3 \mathrm{X} 10 \mathrm{~mL})$. The volatiles were removed by oil pump vacuum to yield a white solid; $1.17 \mathrm{~g} 1.9 \mathrm{mmol}(87 \%)$

${ }^{1} \mathrm{H}$ NMR (300 MHz, DMSO- $\left.d_{6}\right) \delta$ ppm 7.67 and $7.61\left(\mathrm{~d}, J^{3}=1.91 \mathrm{~Hz}, \mathrm{H} 4\right.$ and H5), 4.14 (t, $\left.J^{3}=7.3 \mathrm{~Hz}, 2 \mathrm{H}, \mathrm{NC}_{2} \mathrm{CH}_{2}\right), 3.74\left(\mathrm{~s}, 3 \mathrm{H}, \mathrm{NC} \underline{H}_{3}\right), 2.58\left(\mathrm{~s}, 3 \mathrm{H}, \mathrm{NC}\left(\underline{\mathrm{C}}_{3}\right) \mathrm{N}\right), 1.91-1.58(\mathrm{~m}$, $12 \mathrm{H}), 1.49\left(\mathrm{t}, J^{3}=11.5 \mathrm{~Hz}, 2 \mathrm{H}, 2 \mathrm{CH}_{2} \mathrm{PC} \underline{H}\right), 1.36\left(\mathrm{~m}, 2 \mathrm{H}, \mathrm{CH}_{2} \mathrm{C}_{2} \mathrm{P}\right), 1.16(\mathrm{~m}, 10 \mathrm{H})$.

${ }^{13} \mathrm{C}$ NMR $\left(75 \mathrm{MHz}, D M S O-d_{6}\right) \delta \mathrm{ppm} 144.1\left(\mathrm{~s}, \mathrm{~N} \underline{C}\left(\mathrm{CH}_{3}\right) \mathrm{N}\right), 122.2$ and $120.7(\mathrm{~s}, \mathrm{C} 4$ and C5), 119.4 (q, $\left.J_{\mathrm{CF}}=322.0 \mathrm{~Hz}, \mathrm{NTf}_{2}^{-}{ }^{-}\right), 48.3\left(\mathrm{~d}, J_{\mathrm{CP}}^{3}=14.2 \mathrm{~Hz}, \mathrm{NC}_{2} \mathrm{CH}_{2}\right), 34.5\left(\mathrm{~s}, \mathrm{NC}_{3}\right)$, $32.6\left(\mathrm{~d}, J=13.3 \mathrm{~Hz}, \mathrm{CH}_{2} \mathrm{P} \underline{\mathrm{CH}}\right), 29.7,26.6$, and $26.6(3 \mathrm{~d}, J=14.5 \mathrm{~Hz}, 11.2 \mathrm{~Hz}$, and $7.5 \mathrm{~Hz}$, 3C, Cy), $28.4\left(\mathrm{~d}, J_{\mathrm{CP}}^{2}=8.37 \mathrm{~Hz}, \underline{\mathrm{C}} \mathrm{H}_{2} \mathrm{CH}_{2} \mathrm{P}\right), 17.1\left(\mathrm{~d}, J_{\mathrm{CP}}=18.5 \mathrm{~Hz}, \mathrm{CH}_{2} \underline{\mathrm{CH}_{2}} \mathrm{P}\right), 9.1$ (s, $\left.\mathrm{NC}\left(\underline{C}_{3}\right) \mathrm{N}\right) .{ }^{31} \mathrm{P}$ NMR $\left(121 \mathrm{MHz}, D M S O-d_{6}\right) \delta \mathrm{ppm}-7.6$ (s). ESI/MS(+): m/z calc. for $\left[\mathrm{C}_{20} \mathrm{H}_{36} \mathrm{~N}_{2} \mathrm{P}\right]^{+}$335.2616, found 335.2618.

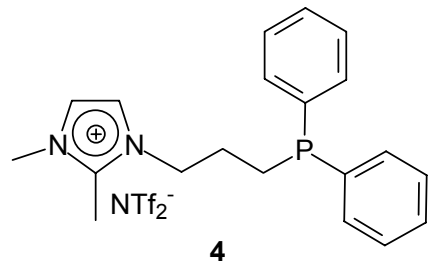

In a drybox, 1-allyl-2,3-dimethyl-imidazolium $\operatorname{NTf}_{2}(0.968 \mathrm{~g}$, $2.32 \mathrm{mmol}), \mathrm{HPPh}_{2}(0.605 \mathrm{~g}, 3.25 \mathrm{mmol})$ and AIBN (8 mg, $0.05 \mathrm{mmol}$ ) were combined in a resealable Schlenk flask. The mixture was stirred at $70^{\circ} \mathrm{C}$ for $48 \mathrm{~h}$; during which time an additional AIBN charge (38 mg, $0.23 \mathrm{mmol}$ ) was added to the reaction mixture. The reaction mixture was washed with ethyl ether $(3 \mathrm{X} \mathrm{10mL})$. The volatiles were removed by oil pump vacuum to yield a viscous liquid; $1.32 \mathrm{~g}(94 \%)$.

${ }^{1} \mathrm{H}$ NMR (300 MHz, DMSO-d $\left.d_{6}\right) \delta$ ppm 7.69 and 7.60 (s, H4 and H5), 7.48-7.30 (m, 10H, $2 \mathrm{Ph}), 4.21\left(\mathrm{t}, J^{3}=6.9 \mathrm{~Hz}, 2 \mathrm{H}, \mathrm{NC}_{2} \mathrm{CH}_{2}\right), 3.73\left(\mathrm{~s}, 3 \mathrm{H}, \mathrm{NC} \underline{H}_{3}\right), 2.54\left(\mathrm{~s}, 3 \mathrm{H}, \mathrm{NC}\left(\underline{\mathrm{C}}_{3}\right) \mathrm{N}\right)$, $2.11\left(\mathrm{~m}, 2 \mathrm{H}, \mathrm{CH}_{2} \mathrm{C} \underline{H}_{2} \mathrm{P}\right), 1.78\left(\mathrm{~m}, 2 \mathrm{H}, \mathrm{NCH}_{2} \mathrm{C}_{2}\right) .{ }^{13} \mathrm{C}$ NMR $\left(75 \mathrm{MHz}, D M S \mathrm{O}-d_{6}\right) \delta \mathrm{ppm}$ $144.2\left(\mathrm{~s}, \mathrm{~N} \underline{C}\left(\mathrm{CH}_{3}\right) \mathrm{N}\right), 137.7(\mathrm{~d}, J=13.3 \mathrm{~Hz}, 2 \mathrm{C}$ ipso- $\mathrm{Ph}), 132.3\left(\mathrm{~d}, J_{\mathrm{CP}}^{2}=18.6 \mathrm{~Hz}, 4 \mathrm{C} o-\right.$ $\mathrm{Ph}), 128.7$ (s, 2C $p-\mathrm{Ph}$ ), 128.5 (d, $\left.J_{\mathrm{CP}}^{3}=6.6 \mathrm{~Hz}, 4 \mathrm{C} m-\mathrm{Ph}\right), 122.2$ and 120.7 (s, C4 and C5), $119.4\left(\mathrm{q}, J_{\mathrm{CF}}=322.3, \mathrm{NTf}_{2}^{-}\right), 48.0\left(\mathrm{~d}, J_{\mathrm{CP}}^{3}=14.9 \mathrm{~Hz}, \mathrm{~N}_{\mathrm{CH}} \mathrm{CH}_{2}\right), 34.5\left(\mathrm{~s}, \mathrm{NC} \underline{H}_{3}\right), 25.9(\mathrm{~d}$, $\left.J=18.2 \mathrm{~Hz}, \mathrm{CH}_{2} \underline{\mathrm{CH}} \mathrm{H}_{2} \mathrm{P}\right), 22.8\left(\mathrm{~d}, J_{\mathrm{CP}}^{2}=11.7 \mathrm{~Hz}, \mathrm{NCH}_{2} \underline{\mathrm{CH}} \mathrm{H}_{2}\right), 9.1\left(\mathrm{~s}, \mathrm{NC}\left(\underline{\mathrm{C}} \mathrm{H}_{3}\right) \mathrm{N}\right) .{ }^{31} \mathrm{P} \mathrm{NMR}$ 
$\left(121 \mathrm{MHz}, D M S O-d_{6}\right) \delta \mathrm{ppm}-16.4$ (s). ESI/MS(+): m/z calc. for $\left[\mathrm{C}_{20} \mathrm{H}_{24} \mathrm{~N}_{2} \mathrm{P}\right]^{+} 323.1677$; found 323.1679 .

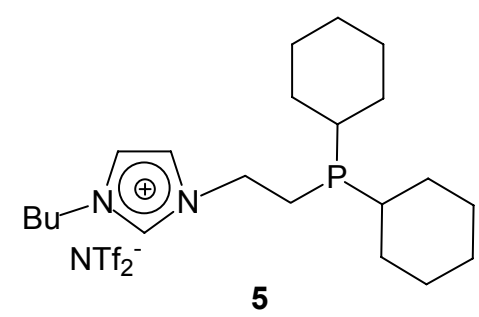

In a drybox, 1-vinyl-3-butyl-imidazolium $\mathrm{NTf}_{2}(0.834 \mathrm{~g}, 1.93$ $\mathrm{mmol}), \mathrm{HPCy}_{2}(0.793 \mathrm{mg}, 4 \mathrm{mmol}), \mathrm{CF}_{3} \mathrm{Ph} 5 \mathrm{~mL}$ and $\mathrm{ABCN}$ (10 $\mathrm{mg}, 0.04 \mathrm{mmol})$ were combined in a resealable Schlenk flask. The biphasic mixture was stirred at $80^{\circ} \mathrm{C}$ for $24 \mathrm{~h} .{ }^{31} \mathrm{P}$ analysis of the reaction mixture shows 3 major species.

${ }^{31} \mathrm{P}$ NMR $\left(121 \mathrm{MHz}, D M S O-d_{6}\right) \delta \mathrm{ppm} 5.6(\mathrm{~s}),-10.8(\mathrm{~s})$ major peaks, $10.31(\mathrm{~s}, 1 \mathrm{C})$ minor peak. ESI/MS(+): m/z calc. for $\left[\mathrm{C}_{21} \mathrm{H}_{38} \mathrm{~N}_{2} \mathrm{P}\right]^{+} 349.2772$, found 349.2777 .

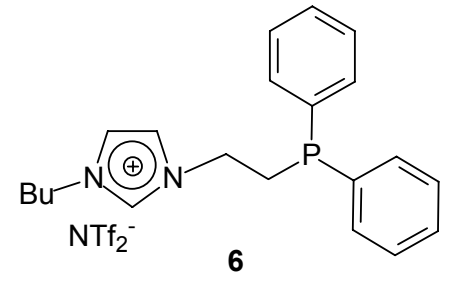

In a drybox, 1-vinyl-3-butyl-imidazolium $\mathrm{NTf}_{2}(0.982 \mathrm{~g}, 2.28$ $\mathrm{mmol}), \mathrm{HPPh}_{2}(0.560 \mathrm{~g}, 3.00 \mathrm{mmol})$ and AIBN $(10 \mathrm{mg}, 0.06$ $\mathrm{mmol}$ ) were combined in a resealable Schlenk flask. The mixture was stirred at $70{ }^{\circ} \mathrm{C}$ for $24 \mathrm{~h}$ yielding a viscous oil that which was washed with ethyl ether $(3 \times 10 \mathrm{~mL})$. Removal of volatiles by oil pump vacuum yields a viscous oil; $1.27 \mathrm{~g}(88 \%)$.

${ }^{1} \mathrm{H}$ NMR (300 MHz, DMSO-d $\left.d_{6}\right) \delta$ ppm 9.21 (s, H2), 7.83 and $7.72\left(2 \mathrm{~d}, J^{3}=1.4 \mathrm{~Hz}, \mathrm{H} 4\right.$ and $\mathrm{H} 5), 7.58-7.27(\mathrm{~m}, 10 \mathrm{H}, 2 \mathrm{Ph}), 4.30\left(\mathrm{~m}, 2 \mathrm{H}, \mathrm{NC}_{2} \mathrm{CH}_{2} \mathrm{P}\right), 4.10\left(\mathrm{t}, J^{3}=7.0 \mathrm{~Hz}, 2 \mathrm{H}\right.$, $\left.\mathrm{NC}_{2} \mathrm{CH}_{2} \mathrm{CH}_{2} \mathrm{CH}_{3}\right), 2.73\left(\mathrm{~m}, 2 \mathrm{H}, \mathrm{NCH}_{2} \mathrm{C}_{2} \mathrm{P}\right), 1.73\left(\mathrm{~m}, 2 \mathrm{H}, \mathrm{NCH}_{2} \mathrm{C}_{2} \mathrm{CH}_{2} \mathrm{CH}_{3}\right), 1.24(\mathrm{~m}$, $\left.2 \mathrm{H}, \mathrm{NCH}_{2} \mathrm{CH}_{2} \mathrm{C}_{2} \mathrm{CH}_{3}\right), 0.90\left(\mathrm{t}, J^{3}=7.2 \mathrm{~Hz}, 3 \mathrm{H}, \mathrm{NCH}_{2} \mathrm{CH}_{2} \mathrm{CH}_{2} \mathrm{C}_{3}\right) .{ }^{13} \mathrm{C} \mathrm{NMR}(75 \mathrm{MHz}$, DMSO-d $\left.d_{6}\right) \delta$ ppm $136.6\left(\mathrm{~d}, J=12.2 \mathrm{~Hz}, 2 \mathrm{C}\right.$ ipso-Ph), $135.9(\mathrm{~s}, \mathrm{~N} \underline{C H N}), 132.3\left(\mathrm{~d}, J_{\mathrm{CP}}^{2}=\right.$ $19.3 \mathrm{~Hz}, 4 \mathrm{C} o-\mathrm{Ph}), 129.0(\mathrm{~s}, 2 \mathrm{C} p-\mathrm{Ph}), 128.6\left(\mathrm{~d}, J_{\mathrm{CP}}^{3}=6.9 \mathrm{~Hz}, 4 \mathrm{C} m-\mathrm{Ph}\right), 122.3$ and 122.2 (2s, C4 and C5), 119.4 (q, $J_{\mathrm{CF}}=321.8 \mathrm{~Hz}, \mathrm{NTf}_{2}{ }^{-}$), 48.4 (s, $\left.1 \mathrm{C}, \mathrm{N}^{-} \mathrm{H}_{2} \mathrm{CH}_{2} \mathrm{CH}_{2} \mathrm{CH}_{3}\right), 46.6$ $\left(\mathrm{d}, J_{\mathrm{CP}}^{2}=25.6 \mathrm{~Hz}, \mathrm{~N}_{\mathrm{CH}} \mathrm{CH}_{2} \mathrm{P}\right), 31.2\left(\mathrm{~s}, 1 \mathrm{C}, \mathrm{NCH}_{2} \underline{C H}_{2} \mathrm{CH}_{2} \mathrm{CH}_{3}\right), 27.1\left(\mathrm{~d}, J_{\mathrm{CP}}=13.7 \mathrm{~Hz}\right.$,

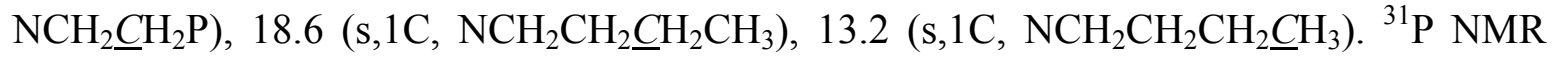
$\left(121 \mathrm{MHz}, D M S O-d_{6}\right) \delta \mathrm{ppm}-21.4$ (s). $\mathrm{ESI} / \mathrm{MS}(+)$ : $\mathrm{m} / \mathrm{z}$ calc. for $\left[\mathrm{C}_{21} \mathrm{H}_{26} \mathrm{~N}_{2} \mathrm{P}\right]^{+} 337.1834$, found 337.1831 . 


\section{$\left(\left(1,3-B i s(M e s) H_{2} I m\right)\right)(3)(C l){ }_{2} \mathrm{Ru}(=\mathrm{CHPh})(8)$}

A Schlenk flask was charged with $\left(\left(1,3-\mathrm{Bis}(\mathrm{Mes}) \mathrm{H}_{2} \mathrm{Im}\right)\right)(\mathrm{Py})_{2}(\mathrm{Cl})_{2} \mathrm{Ru}(=\mathrm{CHPh})(0.178 \mathrm{~g}$, $0.245 \mathrm{mmol}), 3(0.162 \mathrm{~g}, 0.264 \mathrm{mmol})$, and $\mathrm{CF}_{3} \mathrm{Ph}(3.0 \mathrm{~mL})$. The mixture was stirred at room temperature for $15 \mathrm{~min}$. The solvent was removed by oil pump vacuum and the residue washed with pentane $(3 \mathrm{X} 10 \mathrm{~mL})$ to give a pink solid; yield: $0.215 \mathrm{~g}$ (1.82 mmol, $75 \%)$ :

${ }^{1} \mathrm{H}$ NMR (300 MHz, Toluene- $\left.d_{8}\right) \delta$ ppm 20.05 (s, 1H, Ru=CH), 8.07-7.96 (m,1H), 7.87$7.66(\mathrm{~m}, 5 \mathrm{H}), 7.58(\mathrm{~s}, 3 \mathrm{H}), 7.48$ and $7.35(2 \mathrm{~s}, \mathrm{H} 4$ and $\mathrm{H} 5), 4.20\left(\mathrm{t}, J^{3}=5.9 \mathrm{~Hz}, \mathrm{NC}_{2} \mathrm{CH}_{2}\right)$,

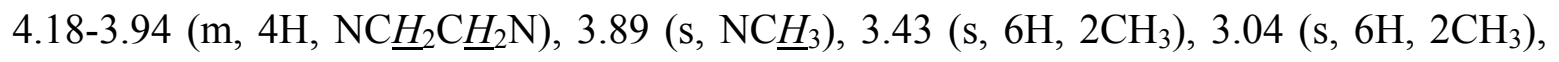
2.90 (s, 3H, $\left.\mathrm{CH}_{3}\right), 2.87$ (s, 3H, $\left.\mathrm{CH}_{3}\right), 2.59$ (s, 3H, $\left.\mathrm{NC}\left(\mathrm{C}_{3}\right) \mathrm{N}\right), 2.56-1.53$ (m, overlap, 28H). ${ }^{13} \mathrm{C}$ NMR $\left(75 \mathrm{MHz}\right.$, Toluene- $\left.d_{8}\right) \delta \mathrm{ppm} 220.5\left(\mathrm{~d}, J_{\mathrm{CP}}=81.8 \mathrm{~Hz}\right), 151.4,143.9,139.1$, $138.3,137.8,136.9,135.4,130.9,130.0,129.4,128.1,122.1,120.8\left(\mathrm{q}, J_{C F}=321.6 \mathrm{~Hz}\right.$, $\left.\mathrm{NTf}_{2}{ }^{-}\right), 120.9,51.47\left(\mathrm{~d}, J_{C P}=78.56 \mathrm{~Hz}, 1 \mathrm{C}\right), 51,9,50.9,49.2\left(\mathrm{~d}, J_{C P}=13.3 \mathrm{~Hz}\right), 34.6,33.0$ $\left(\mathrm{d}, J_{C P}=18.0 \mathrm{~Hz}\right), 28.8\left(\mathrm{~d}, J_{C P}=23.6 \mathrm{~Hz}\right), 27.8\left(\mathrm{~d}, J_{C P}=11.9 \mathrm{~Hz}\right), 27.5\left(\mathrm{~d}, J_{C P}=8.7 \mathrm{~Hz}\right)$, 20.3, 18.7, 9.6. ${ }^{31} \mathrm{P}$ NMR (121 MHz, Toluene- $\left.d_{8}\right) \delta \mathrm{ppm} 26.6(\mathrm{~s}) . \mathrm{ESI} / \mathrm{MS}(+): \mathrm{m} / \mathrm{z}$ calc. for $\left[\mathrm{C}_{48} \mathrm{H}_{69} \mathrm{Cl}_{2} \mathrm{~N}_{4} \mathrm{PRu}\right]^{+}$904.3688, found 904.3685.

\section{Typical procedure for the RCM catalytic reactions}

In a glove box, a two-neck Schlenk flask was charged with 8 (0.0059 g, $0.0050 \mathrm{mmol})$ and $\mathrm{BMI} \mathrm{PF}_{6}(1.5 \mathrm{~g})$. A second Schlenk flask was charged with the appropriate substrate (2.0 $\mathrm{mmol})$ and toluene $(4 \mathrm{~mL})$. After the IL catalyst solution reached the desired temperature, the substrate solution was added in one portion against a stream of argon. Samples were periodically removed by syringe for GC and GC-MS analyses. After the reaction, the upper organic phase was separated and toluene removed under vacuum. A fresh charge of substrate was added to the IL phase. Spectral characteristics of RCM products were in agreement with previously reported data.

Cyclohexene. MS (EI): m/z (\%): 82(45), 67(100), 54(62), 41(24)

Cyclopent-3-ene-1,1-dicarboxylic acid diethyl ester. ${ }^{1} \mathrm{H}$ NMR $\left(300 \mathrm{MHz}, C D C l_{3}\right) \delta \mathrm{ppm}$ $5.61(\mathrm{~s}, 2 \mathrm{H}), 4.20(\mathrm{q}, J=7.11 \mathrm{~Hz}, 4 \mathrm{H}), 3.01(\mathrm{~s}, 4 \mathrm{H}), 1.25$ (t, $J=7.12 \mathrm{~Hz}, 6 \mathrm{H})$. MS (EI): m/z (\%): 212(30), 166(52), 138(100), 111(50), 93(45), 79(58), 66(64) 
3-Methyl-cyclopent-3-ene-1,1-dicarboxylic acid diethyl ester. ${ }^{1} \mathrm{H}$ NMR $(300 \mathrm{MHz}$, $\left.C D l_{3}\right) \delta \operatorname{ppm} 5.19(\mathrm{~m}, 1 \mathrm{H}), 4.19(\mathrm{q}, J=7.11 \mathrm{~Hz}, 4 \mathrm{H}), 2.96(\mathrm{~m}, 2 \mathrm{H}), 2.90(\mathrm{~s}, 2 \mathrm{H}), 1.71(\mathrm{~s}$, $3 \mathrm{H}), 1.25$ (t, $J=7.12 \mathrm{~Hz}, 6 \mathrm{H})$. MS (EI): m/z (\%): 226(27), 181(18), 152(100), 124(28), 107(42), 79(58).

3,4-Dimethyl-cyclopent-3-ene-1,1-dicarboxylic acid diethyl ester. MS (EI): m/z (\%): 240(19), 167(30), 166(100), 121(27), 93(51)

(1) Necas, D.; Tursky, M.; Kotora, M. Journal of the American Chemical Society 2004, 126, 10222-10223.

(2) Sanford, M. S.; Love, J. A.; Grubbs, R. H. Organometallics 2001, 20, 53145318.

(3) Cassol, C. C.; Ebeling, G.; Ferrera, B.; Dupont, J. Advanced Synthesis \& Catalysis 2006, 348, 243-248. 


\section{Spectra}
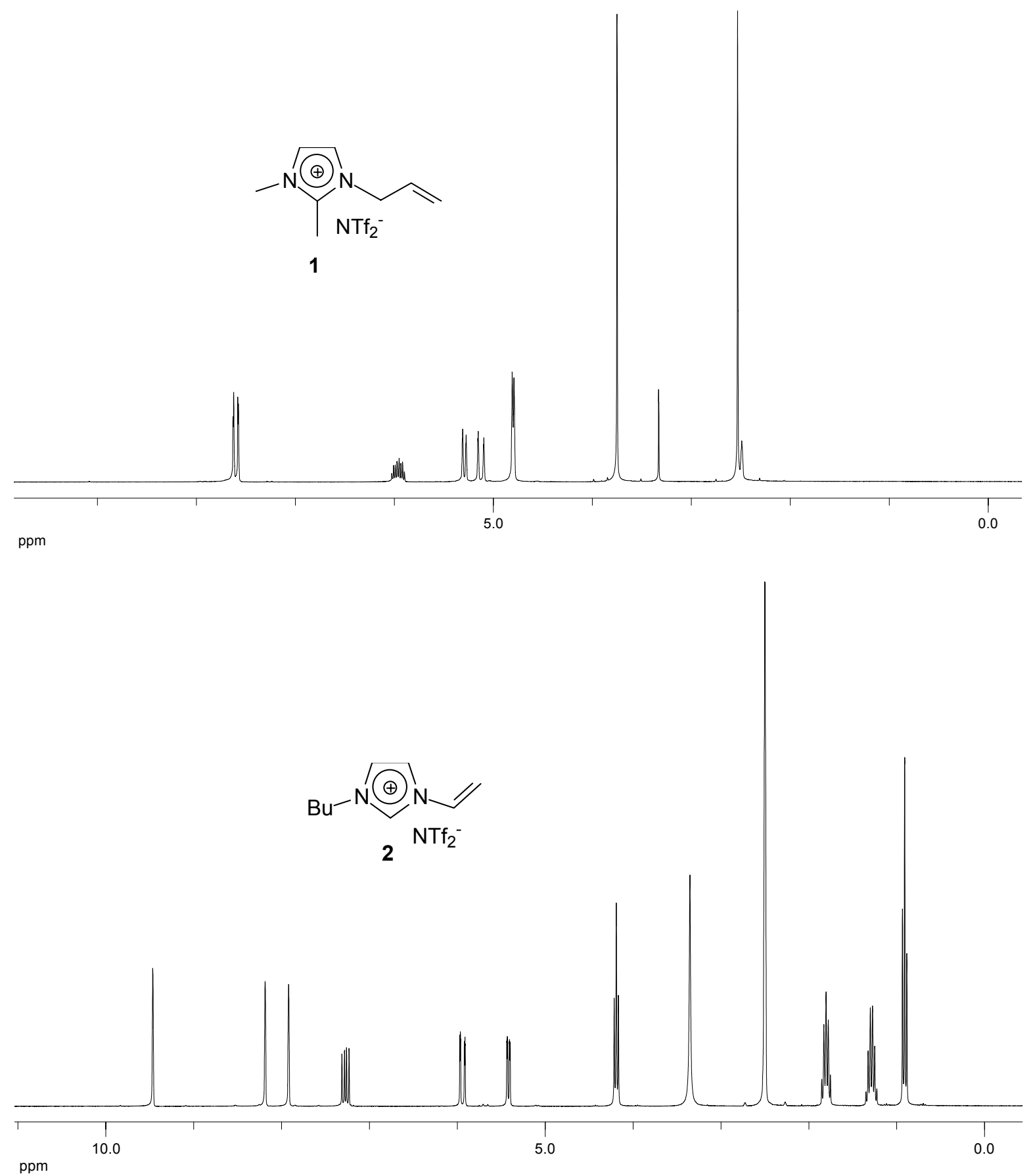

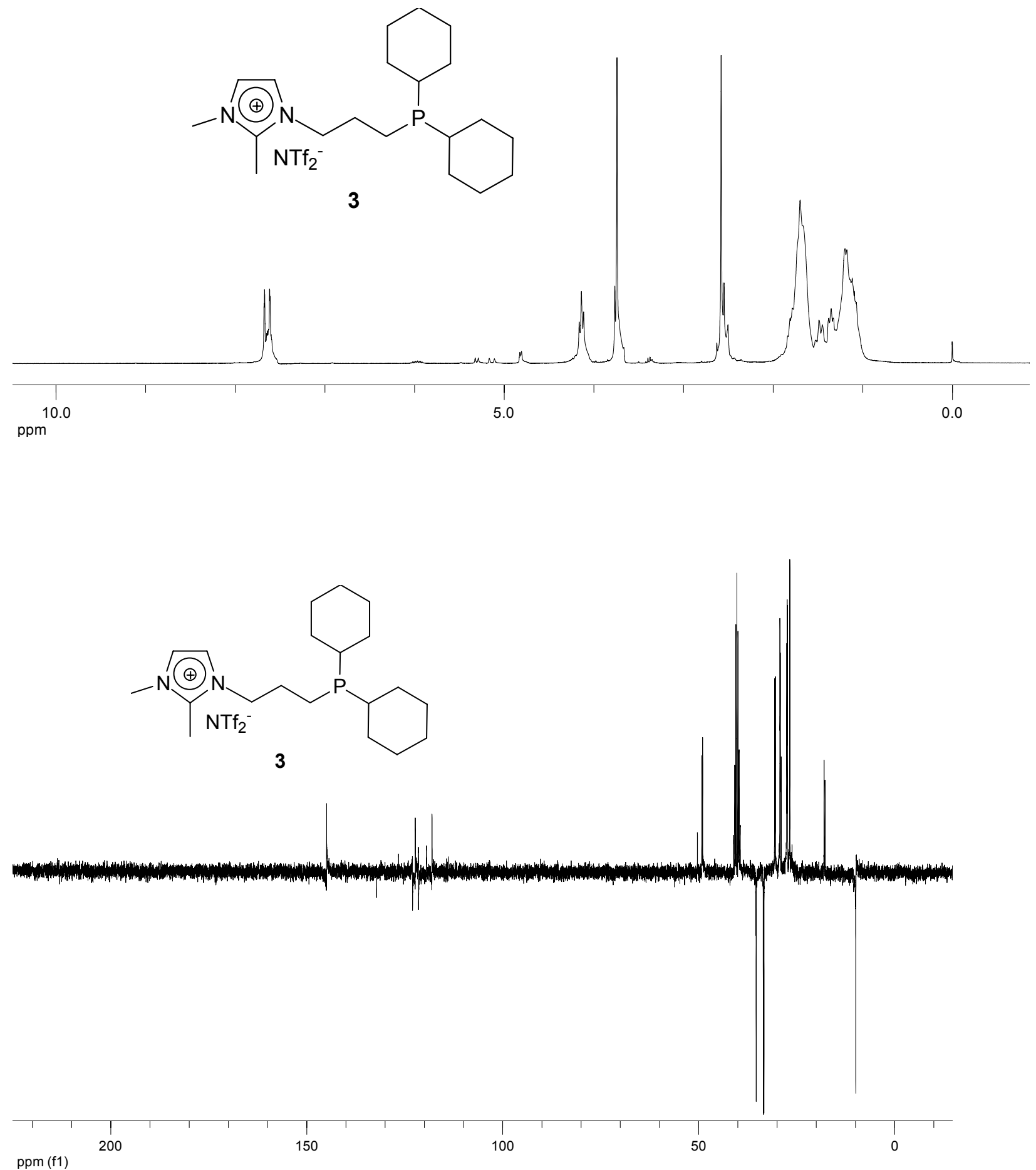

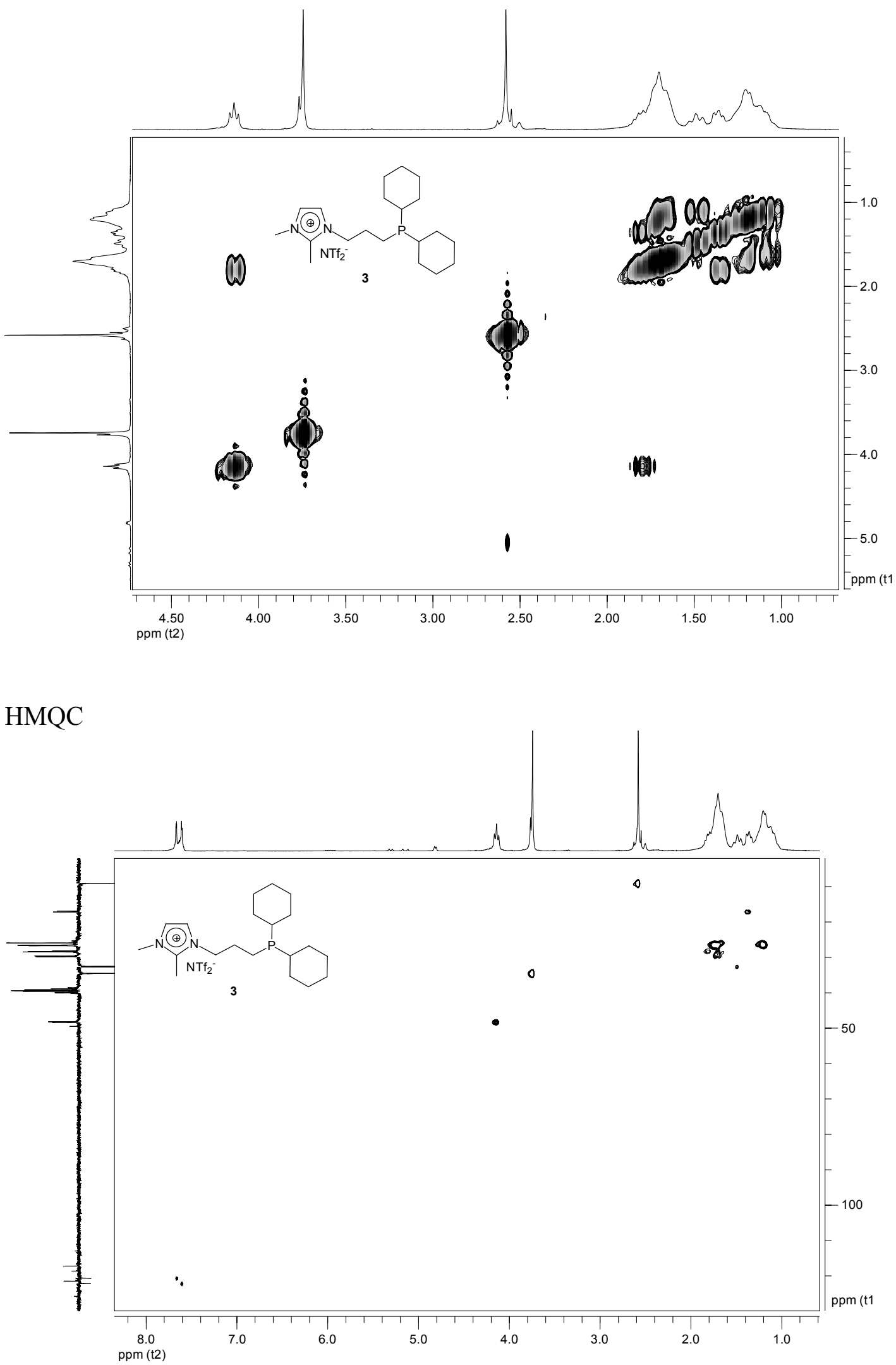

S9 


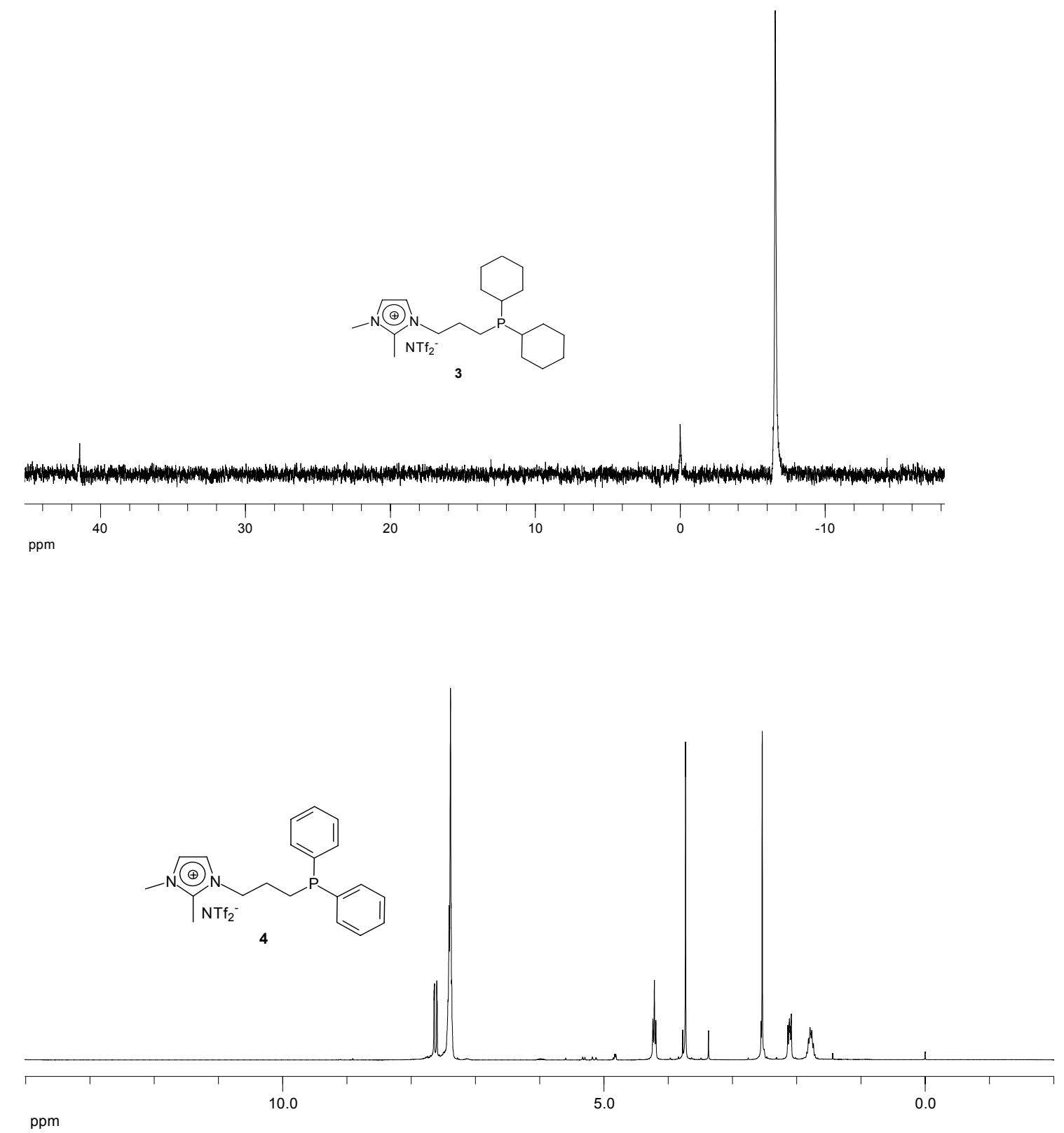



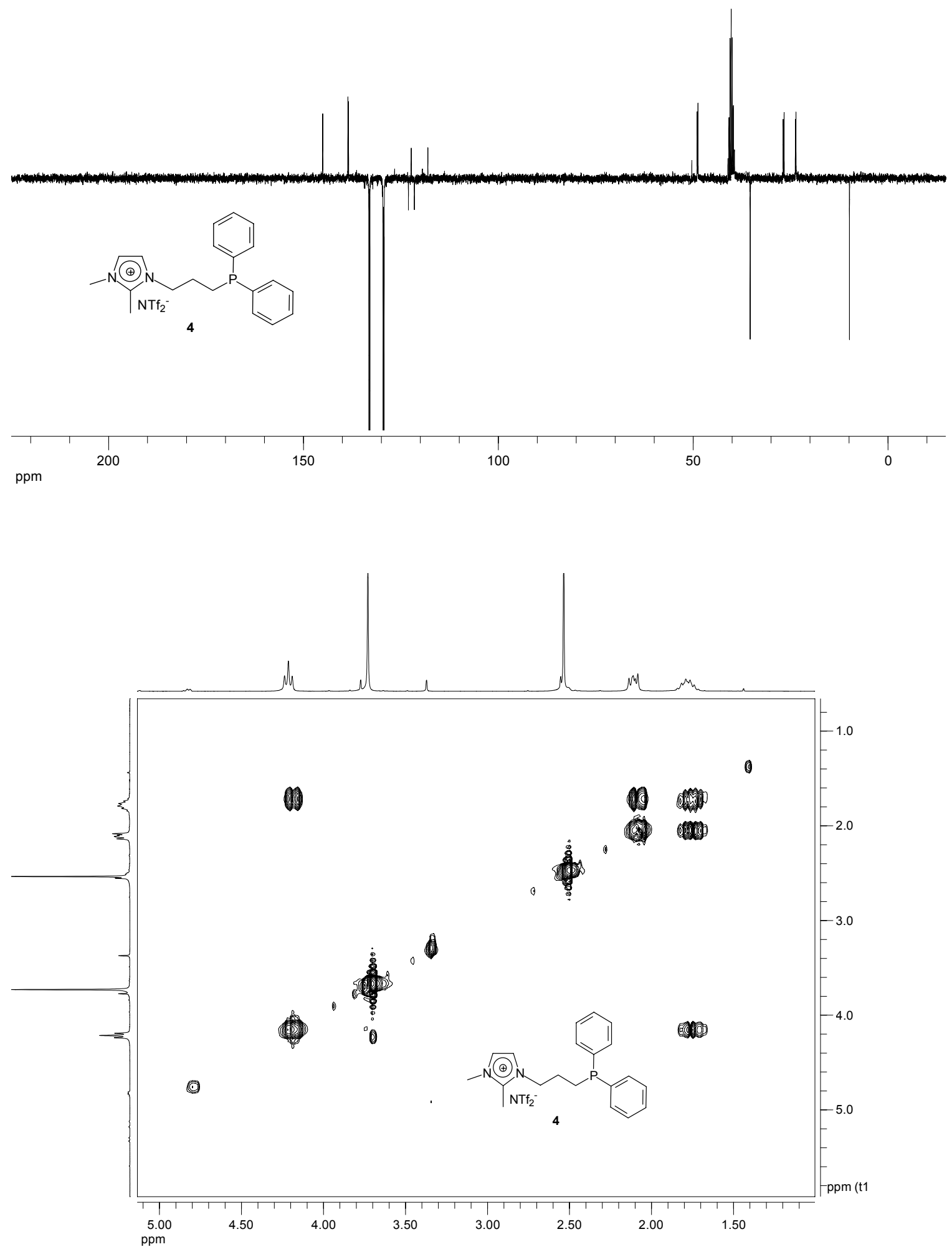

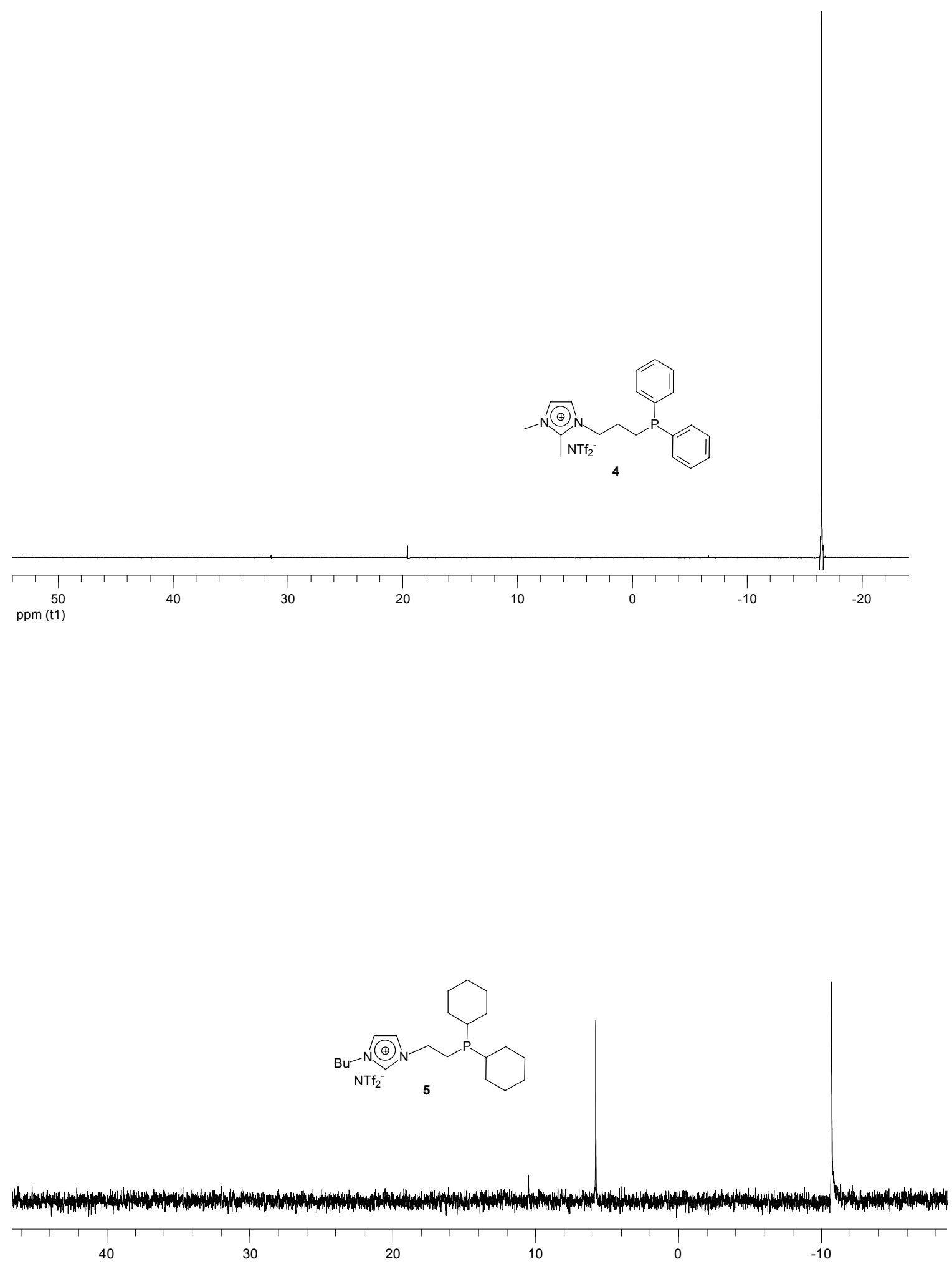

ppm (t1)

S12 

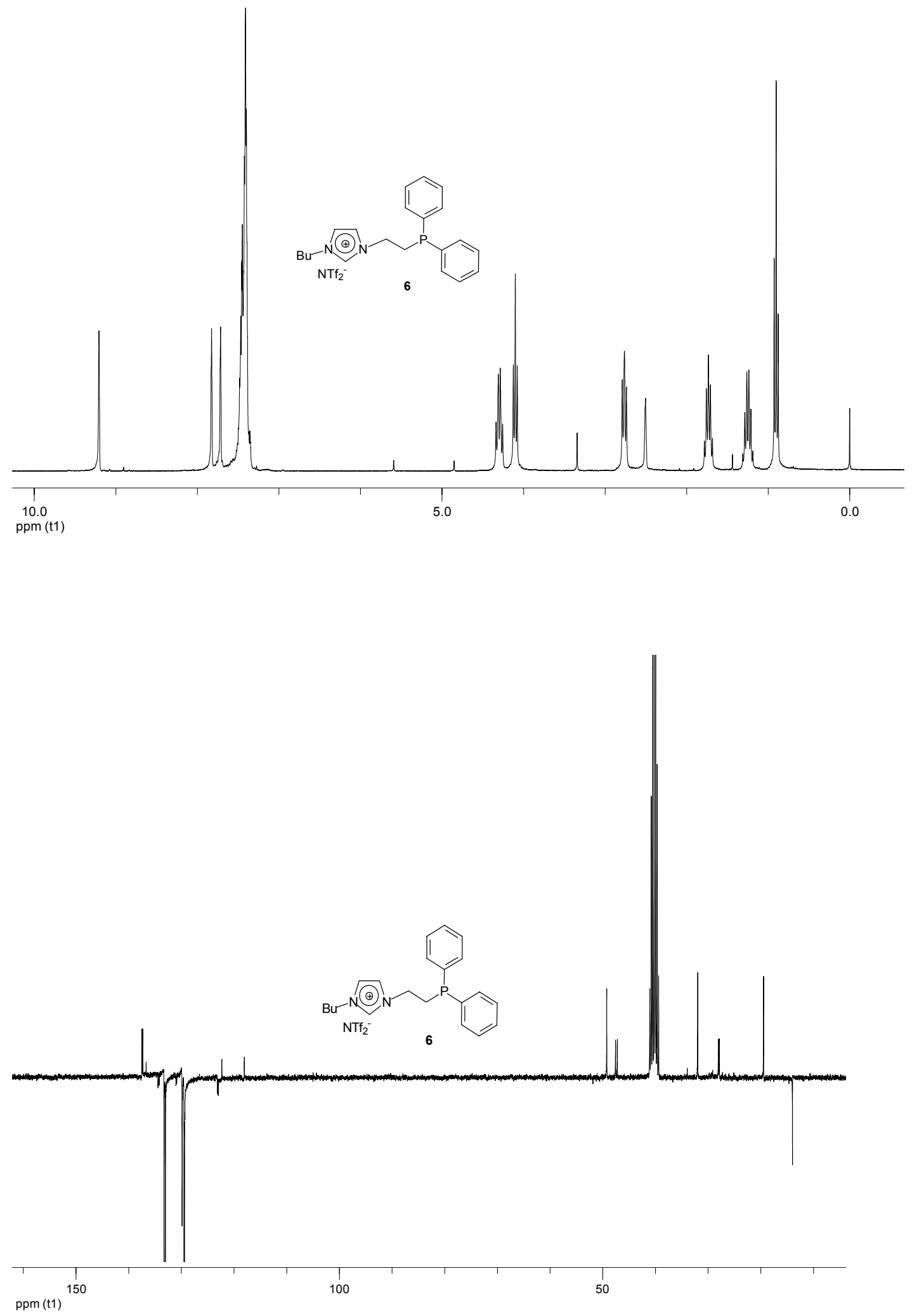

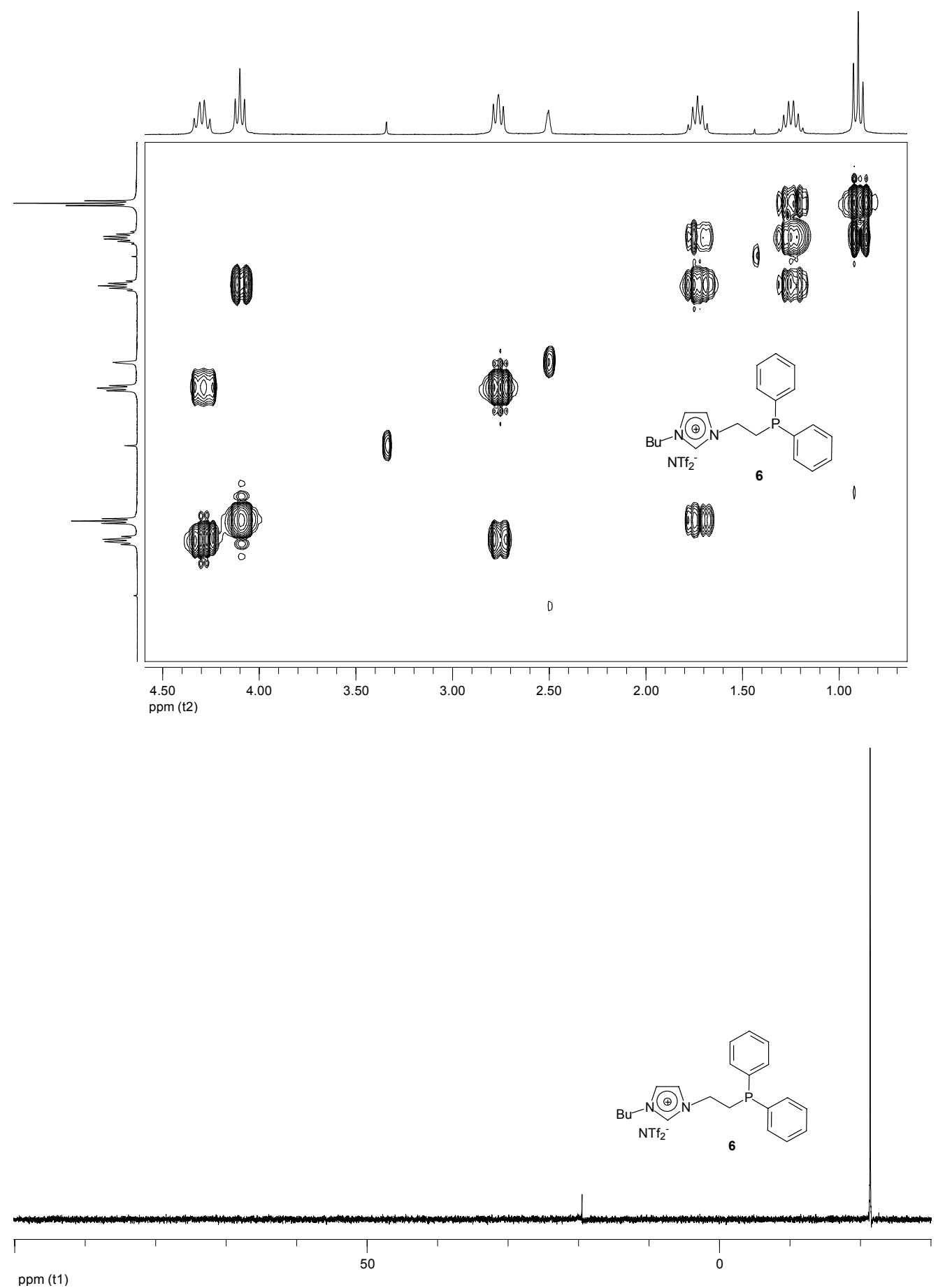

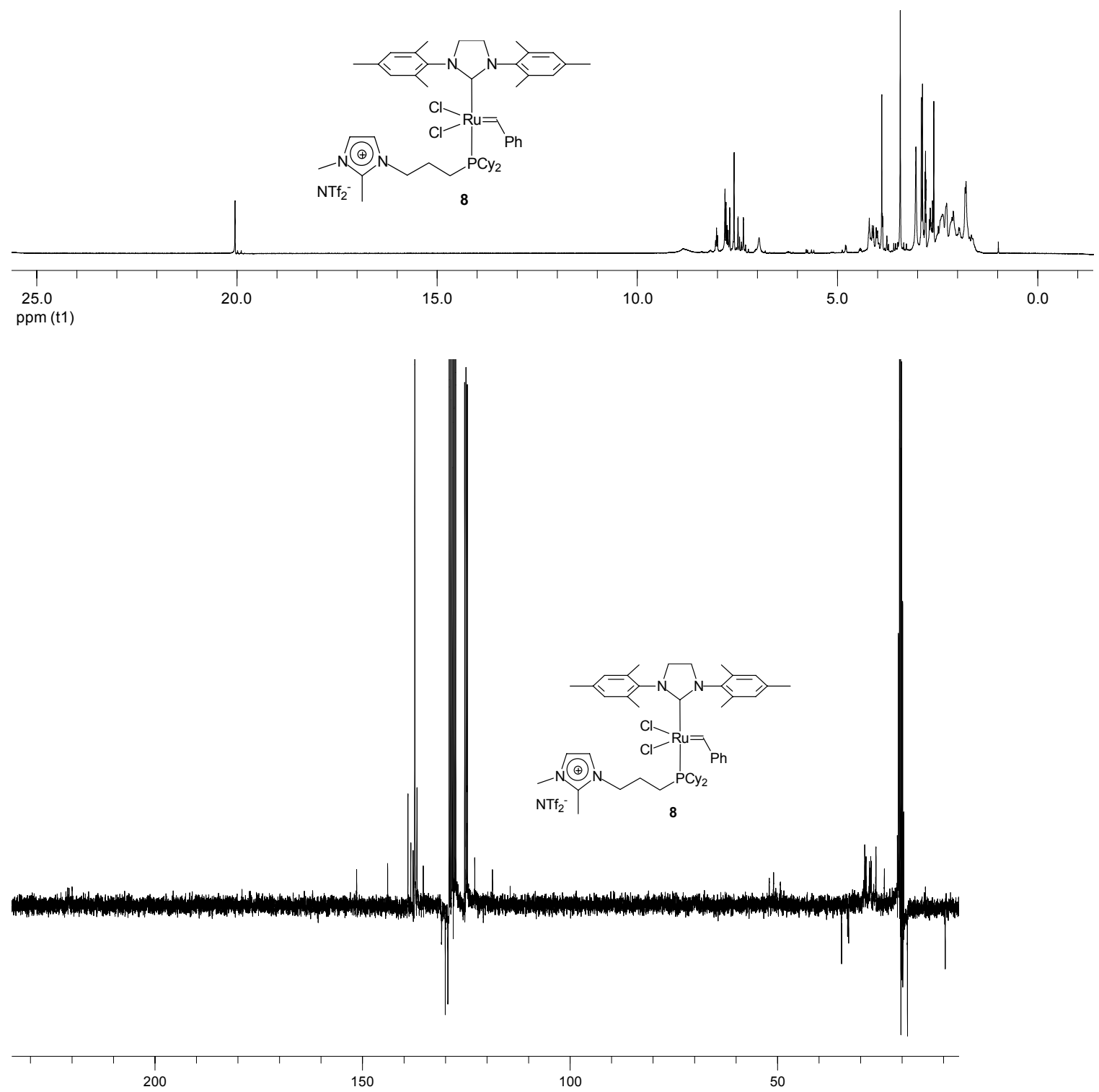

ppm (t1)

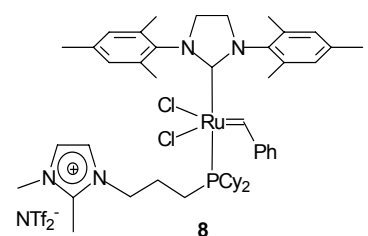

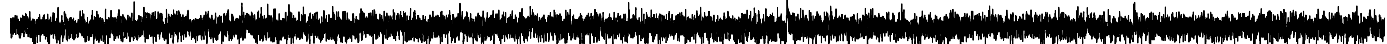

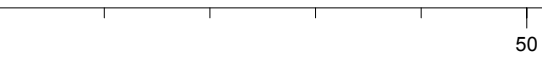

ppm (t1) 